\title{
Towards a Medical Imaging Standard Capture and Analysis Software
}

\author{
by R. Vardasca***, P. Plassmann**, J. Gabriel* and E. F. J. Ring** \\ * LABIOMEP, IDMEC-FEUP Campus, Faculty of Engineering, University of Porto, Porto, Portugal, \\ ricardo.vardasca@fe.up.pt, jgabriel@fe.up.pt \\ ${ }^{* *}$ Medical Imaging Research Unit, Faculty of Computing, Engineering and Science, University of \\ South Wales, Wales, United Kingdom, ricardo.vardasca@southwales.ac.uk, \\ peter.plassmann@southwales.ac.uk, francis.ring@southwales.ac.uk
}

\begin{abstract}
Medical thermal imaging offers real time physiology monitoring, providing important information about the microcirculatory and autonomous nervous systems. The recording equipment has evolved over times, presenting actually very good definition, sensitive and image quality. Image capture procedures have been developed, published and tested. The use of computers and software facilitates the understanding of the images, although the current scenario in medical application is that there is no specific camera or software package designed and developed specifically for clinical use. This work outlines the features that a capture and analysis system needs to have to support the medical imaging modality.
\end{abstract}

\section{Introduction}

Medical thermal imaging is a non-invasive medical screening modality that maps human body surface temperature providing real-time physiology monitoring of cutaneous microcirculation and autonomous nervous system. Ray Lawson [1], a Canadian Physician, to study surface temperature of breast tumors, has introduced this medical imaging modality in 1956. It has been applied clinically in inflammatory arthritis, osteoarthritis, soft tissue rheumatism, enthesopathies, tennis elbow, fibromyalgia, complex regional pain syndrome, peripheral circulation, fever screening, burns, renal dialysis and malignant diseases [1,2].

The equipment's suffered important developments since have been introduced in the medical field, the cameras evolved in a reduction of size, becoming more portable and reliable. Despite in early days, where good definition images were only possible through liquid nitrogen refrigeration, using larger and noisy equipment, actually the uncooled infrared cameras have similar image quality when compared with the cooled. The sensors had also considerable improvements, which allowed a reduction in size and a rise in their sensitivity [3].

In order to verify if the recording equipment is under adequate conditions, for quality assurance purposes, a battery of simple tests was proposed to verify the following parameters: start-up drift, long-term offset drift, offset variation over observed range, image non-uniformity, thermal 'flooding', spatial resolution and thermal resolution. These tests are recommended and should be performed periodically in order to make sure that the measurements being made are correct [4].

Despite the early interest from the clinical professionals in the modality and equipment limitation, good studies were performed [1]. However in 1977, the Breast Cancer Detection Demonstration Project (BCDDP) report was published about the sensitivity of thermography compared to other methods of breast cancer detection. The results demonstrated that thermography appeared in third, after ultrasound and mammography, presenting a sensitivity of only $39 \%$ and a specificity of $82 \%$ [5]. This study, using limited equipment and not mentioning any protocol to deal with known conditional environment variables (ambient temperature, humidity, air flow and subject acclimatization period) affecting the examination, has discredited thermography in clinical setting for years.

In order to raise interest and prove the feasibility for the usage of thermal imaging in medicine, concerns were outlined to standardize the capture of images [6 - 8]. Guidelines were proposed for the subject (before and during appointment), equipment and lab preparation, and manner of conducting the examination $[9,10]$. Minimal requirements and specifications for thermal camera suitable for clinical use were outlined [11] and international standards released defining the usage of thermal cameras for massive fever detection [12] and for the recommended mode of deployment including the testing of the systems and the training of its users [13].

All this recent developments contributed to an important improvement of the technique and acceptance from the clinical professionals, however, work needs to be done for standardizing the images analysis.

Ledley [14] introduced the usage of computers in medicine in 1959, since then computers evolved over time, becoming smaller and powerful. Computers in medical thermal imaging are an important tool for image acquisition and 
analysis. Some authors $[15,16]$ designed a decade ago a set of specific requirements needed for dealing with medical infrared images acquisition, storage and specific analysis.

The aim of this research is to outline the minimal requirements for fulfilling the existent gap of having a specific dedicated software for medical thermography support, incorporating the most recent developments in technology, which could make a difference in the wide acceptance and common daily usage of the technique in clinical practice.

\section{Methodology}

Following the minimal requirements for IR cameras that can be used in medical settings [11] a search within the IR cameras manufacturers was performed and the correspondent software's offered were searched and registered along with their offered features.

In the search it was taken into account the requirements of the capture and analysis guidelines, the suggested particularities for medical IR analysis software $[15,16]$, the latest development in image processing and the features offered by the proprietary software packages.

The method used to reach the needed information was the contact with Infrared seller representatives and the Internet search using the well-known Internet browser Google. A table was filled with the most important information and a comparison was performed with the goal of a defining of a group of components that a future standard software package dedicated to medical thermal images capture and analysis needs to accommodate.

\section{Results}

All infrared camera manufacturers provide proprietary software, which allow camera connection (through Firewire, USB, Ethernet or Gigabit-Ethernet and Wi-Fi), interaction with camera commands (focus, temperature scale, emissivity value and recording), image recording in a proprietary format and basic analysis tool. Thermal cameras used in medicine are standard cameras that are also used in industrial and other applications. In consequence the developed proprietary software is also very generic and does not suit the majority of specific clinical applications.

A research group in the University of Glamorgan (actually University of South Wales) [17], UK, had developed over 10 years a computational system, CTHERM ${ }^{\circledR}$, that was able to acquire a thermal images, store and perform analysis and reporting. The software allowed to draw regions of interest to analyze thermal properties, showing the histogram, such as: mean, maximum, minimum temperature and standard deviation. It also permitted to use isotherms and cross-section for more accurate analysis. As a built-in medical prognosis tool it had a specific cold stress test to the hands analysis. Although it only worked in Microsoft Windows Operating Systems 2000 and XP professional and used a FLIR Thermovision SDK®.

Currently FLIR, the major manufacturer of Infrared cameras, provides free and licensed software [18]. The freeware software provided by FLIR are: FLIR QuickReport $₫$, FLIR Tools $₫$, FLIR Tools Mobile $₫$, FLIR Viewer app $₫$ and FLIR Web Viewer ${ }^{\circledR}$. While the provided licensed software are: FLIR Camera Player $\AA$, FLIR IR Monitor ${ }^{\circ}$, FLIR Reporter Pro®, FLIR ThermaCam Researcher Pro®, FLIR ResearchIR Max®, FLIR Tools+®, FLIR Thermovision SDK®, Altair and Talon Ultra®.

The FLIR QuickReport ${ }^{\circledR}$ is a free Windows based application that allows users to organize and analyze the radiometric images from its infrared cameras and present them in a report.

The FLIR Tools $®$ are available for Microsoft Windows ${ }^{\circledR}$ and Apple Mac OS $X ®$ operating systems, and is a free package that allows: importing of infrared images through a USB cable from the camera or SD card; edit radiometric images to thermal tune level and span, change the palette, or adjust parameters such as emissivity and reflective temperature; add measurement tools such as spots, area boxes, circles, lines, Delta T; add text annotations and edit image descriptions; create professional PDF image sheets and reports; add headers, footers, and logos; create, import, edit and export template, choose a report format; edit MSX images and "Sketch on IR/Visual" images; and display stored compass and GPS information.

The FLIR Tools Mobile $®$ is an intuitive application for Android phones and tablets for importing, analyzing, managing, and sharing thermal images that allows wireless connection to FLIR's new T620, T640, and E-Series (E40, E50, and E60) infrared cameras.

FLIR Viewer ${ }^{\circledR}$ is an intuitive app for importing, analyzing, managing, and sharing thermal images that allows wireless FLIR cameras connection through a iOS $®$ phone or tablet. Images can be easily imported and edited, color palettes can be changed and reports can be generated instantly.

The FLIR WebViewer® is free and intuitive and easy-to-use online tool to analyze and evaluate infrared images. Images can be uploaded, then execute simple procedures such as measurements in regions of interest, palettes changing, read out camera properties and save the processed images into the computer. 
The FLIR IR Camera Player ${ }^{\circledR}$ is a free Windows-based remote control and viewer for cameras from FLIR Systems. With it is possible to: record a video stream from the camera; save a frame from the video stream as a snapshot image ( $\left.{ }^{*} . \mathrm{bmp}\right)$; perform autofocus, focus far, and focus near; auto adjust the camera image; freeze the camera image; save a camera image in the camera; change color palette; and add an image description and a text comment to an image.

FLIR IR Monitor $囚$ is a software that connects to all FLIR A32X series products, enabling the user to set up and control of up to nine FLIR A32X cameras simultaneously for simple automation and safety applications. FLIR Reporter Pro $\AA$ is a powerful program for creating compelling, professional, fully customized, easy-to-interpret maintenance reports. Professional Report Wizard allows step-by-step features in combining all IR inspection data (infrared and visual images), temperature measurements, and text notes into a professional and easy-to-interpret report.

FLIR ThermaCam Researcher Pro® is a robust software package with real-time digital storage, measurement, and analysis features. It is extremely versatile, digitally stores and retrieves static and real-time infrared images, live IR digital video sequences, dynamic high-speed events and data directly from the FLIR IR camera allowing in-depth and precise analysis of thermal events. Has built-in measurement functions, providing fast and extensive temperature analysis including: isotherms, spot measurements, line and area measurements, and custom formulas, it is possible to add up to 100 individual measurement tools to a single IR image. Line profiles and histogram charts can be created for more in-depth analysis of area and line tools. Object parameters such as emissivity, distance, reflected temperature, can be modified, even after an image or sequence has been stored to disk. All measurement tools allow independent emissivity and distance settings.

FLIR ResearchIR Max® is a commercial windows-based scientific and professional tool for camera system command and control, high-speed data recording, real-time or playback analysis, and reporting. It allows: sizable image display with selectable color/grey scale palettes; multiple analysis tools with user defined settings for detailed data examination; to save, locate, or open previously stored images or image sequences; multiple image scaling modes with built in histogram display to simplify manual scaling of the image, view detailed image quantitative data from the image analysis tools; and to plot the desired analysis tool result over a period of time or frame number.

The FLIR Tools $+\circledR$ is similar to its freeware version adding extra features such as: pair and group FLIR thermal images and digital photos independent of when or how the separate images were originally captured; stitch FLIR infrared images regardless of the order they were taken to help to measure and paint the complete thermal picture in one full scene (with a minimum of $30 \%$ overlap); record and replay thermal video sequences, create a temporal plot with the recording, and export the sequence to AVI format; and advanced reports with improved speed, allowing to create a Microsoft Word® report $50 \%$ faster than the freeware version.

FLIR Thermovision SDK® is an ActiveX component that gives Windows Visual Basic/C++ programmers an excellent tool to control access and manipulate image data from FLIR infrared cameras. The SDK will accelerate any application programming with FLIR Systems infrared cameras. It allows end users or a system integrator that wants to build its own system utilizing infrared cameras for any purpose.

The ThermoVision SDK allows fully exploitation of the possibilities that modern infrared camera technology offers. There is a version of SDK for National Instruments LabView ${ }^{\circledR}$ development also.

The Altair software package offers powerful and sophisticated features for scientists and engineers to acquire, display and process infrared images, its main features are: exclusively dedicated to High-End thermography, operates with all FLIR High-End cooled infrared cameras, unique temperature analysis functions, real time storage of images on computational device at full frame rate, built-in video player for multi sequence films, full exportation capabilities, filtering management capabilities, external analog signal acquisitions, detector of raw data availability and customized calibration.

The Talon Ultra® software provides connection to old Indigo cameras and offers acquisition of image data of consecutive frames at full camera speed; usage of computer system memory for data acquisition; full-featured data display, reduction, and analysis capability; support to a powerful Visual Basic-compatible macro language for automating data collection, analysis, and reporting; and can be called by Visual Basic or Visual C++ programs;

The TESTO IRSoft $\circledast$ is a windows-based software package that allows TESTO thermal camera connectivity and basic analysis through regions of interest based in lines, rectangles and ellipses [19].

Fluke SmartView ${ }^{\circledR}$ IR Analysis and Reporting Software is a windows-based software that provides a suite of tools for viewing, optimizing, annotating, and analyzing thermal images and videos from a Fluke thermal camera. It also generates fully customizable and professional-looking reports, there is also an app version of this software available for mobiles systems using iOS $₫$ operating system [20].

InfraTec's state-of-the art IRBIS $3 \circledR$ software package is a windows-based tool for thermographic image data analysis and for creating reports in Microsoft Word®. It offers basic functionalities such as: support for infrared camera file formats of InfraTec's product range; multi-lingual user interface; visualization of thermal images with screen/printeroptimized color palettes; manual and automatic temperature range selection; temperature profiles along any lines and across any measured areas; automatic indication of maximum and minimum temperature mean value; print and export of 
thermal images or tables of measured values; display of up to 10 colored isotherms; image improvement through digital filtering; and an integrated Word-based report function [21].

Xenics Xcontrol ${ }^{\circledR}$ is a graphical user interface that allows for easy control of Xenics infrared cameras and acquisition of images. The user can optimize all IR camera control settings such as integration time, AD settings, detector temperature and gain. Moreover a 2-point correction procedure (gain and offset correction, including bad pixel replacement) is available. XControl can also be used to save images and movies in various file formats.

Xenics Xeneth ${ }^{\circledR}$ and Xenics Xeneth Radiometric ${ }^{\circledR}$ are software packages that allow connection to Xenics infrared camera and offers features such as: image live view, storage of digital pictures/movies, image histogram, line profiles, spot meters, time profiles, identification of hot/cold spots and false color mode with various color palettes.

Xenics also offers to their costumers the Xenics Xeneth SDK®, which is an interface for end user development of their own applications to fully operate with Xenics cameras, offering integration for Microsoft Windows $®$ systems with $\mathrm{C} / \mathrm{C}++, \mathrm{C \#}$, VB.Net and Delphi technologies, for Linux systems operability with $\mathrm{C} / \mathrm{C}++$ programming languages and for National Instruments LabView ${ }^{\circledR}$ development, for all the three platforms there are sample code and documentation available [22].

Spectron IR Software ${ }^{\circledR}$ offers conection to Spectron IR cameras, is a FDA approved software package that allows: camera control, false color scales usage, spot measure, basic image processing and reporting [23].

The Generaltools GTi IR Report® operates with GTi and Omega IR cameras and offers features such as: camera control, use of different color scales, spot measurements, basic image processing and reporting [24].

The DIAS Infrared PYROSOFT professional ${ }^{\circledR}$ is a software package that allows connection with DIAS infrared cameras, providing: data acquisition from a DIAS infrared camera; open and edit measured data and sequences; multi document interface for different documents; Bitmap, video and text export; online data saving and bitmap export; definition of regions of interest (ROI): points, lines, rectangles, circles/ellipses and polygons; definition of values of interest (VOI) with alarm calculation, histogram, trend chart and analog/digital value output via IO sub system; difference image and isotherm display; and generation of multi reports for Microsoft@ Word by the integrated reporting function [25].

The TROTEC Thermo Software IC Report Professional ${ }^{\circledR}$ allows connection with TROTEC ICV infrared cameras and offers features of false color image usage, spot measure, basic image processing and reporting [26].

The IRCameras Thermography Suite $\AA$ provides connection with the IRC infrared cameras with thermographic analysis, storage and reporting features [27].

The Mikroptik Smart-eye IRSee Report® allows communication with Omega and Microptik infrared cameras, presenting as features: manage IR images, adjust level, span and color palette, performing measurements from spots, lines, areas, change isotherms and temperature levels, adjust parameters, add text comments and produce reports for Microsoft Word [28].

The Mikron Infrared has 3 software tools for infrared imaging: MicroView®, MikroSpec $®$ and MicroSpec RT® [29]. All the three softwares provide connection to Nec Avio and Satin cameras and operate under Microsoft Windows ${ }^{\circledR}$ operating systems.

The MikroView $®$ offers: move, copy and delete images between camera and computer, read temperature information from any NEC infrared image, read file names, creation dates, camera type from infrared images, change temperature level and span, change color palette, save as Bitmap, copy and paste to other Windows application.

The MikroSpec® allows: advanced thermal image analysis including image calibration, filtering, statistical analysis, and ROls, functions for visual images, voice annotation and text comments, export to MS applications e.g. Word $^{\mathrm{TM}}$, Excel ${ }^{\mathrm{TM}}$ or PowerPoint ${ }^{\mathrm{TM}}$, thermal image sequence reply with direct access video controls, integration of data into a MS Word ${ }^{\mathrm{TM}}$ document, lens calculator, report writer, image converter and sequence converter.

The MicroSpec RT® presents as extra features: real-time image display and analysis; camera remote control functionality; up to $60 \mathrm{fps}$ acquisition using Firewire or $1 \mathrm{fps}$ via Ethernet; 32 movable/resizable ROI with Minimum, Maximum, Average Temperatures and Alarm functions; point, line, broken line, free line, circle, annulus, rectangle, rotated rectangle, polygon, and region, a custom formula ROI type is also available which allows temperatures to be computed using typical Excel TM formulas; convert function for BMP and AVI formats; and optional I/O modules for process control/machine vision.

Independently from the manufacturers there are other software solutions such as med-hot TotalVision ${ }^{\circ}$, Grayess IRT cronista $®$, Grayess IRT Analyzer $®$, Grayess IRT Stitch $\circledast$ and Medicore IRIS-XP®.

The med-hot TotalVision $®[30]$ is a specific medical software package approved by the US Food and Drug Administration (FDA) that uses integrated FLIR SC cameras offering: positional overlays prompt the thermographer to position the patient in the expected position every time, anatomical overlays to further visualize the anatomy associated with the thermal patterns, thumbnail images to offer a quick overview and easy access to captured images, spot readings at the location of the cursor for a quick temperature assessment. On capture: upload for interpretation at the imaging screen, automatic focus, digital zoom, auto saving after each image and progressive movement to the next image 
position. On view screen: color coded drawing tools for temperature analysis, full functions included in the capture software without the capture control panel, opens without engaging the camera, ability to open multiple Windows and reporting.

The Grayess IRT cronista ${ }^{\circledR}$ is a professional thermographic analysis and reporting tool for researchers that provides: accurate detailed analysis including sequence analysis (different types of ROls and statistics), quickly made eye-catching reports in Microsoft Word $\circledast$, and has integrated communication for real-time image acquisition, analysis and process control.

The Grayess IRT Analyzer $\AA$ is a thermographic organizer analyzer and reporter tool that is also available in a pocket PC version and offers: quick and easy one-step report generation in standard Microsoft Word $®$ format for easy exchange of reports with colleagues and customers; detailed analysis with powerful temperature analysis tools: multi points, multi profile lines, area analysis, hot spot detection and many more; analysis on several infrared images simultaneously with adjustable zoom in analysis mode; easy linking of infrared image, visual image, voice and text notes in an automatic or manually manner; easily organize your infrared images: quick overview of infrared image, corresponding visual image and text notes; compatible with a wide variety of infrared image formats, customized report layout templates; and different language settings available.

The Grayess IRT Stitch $®$ is a thermographic image builder tool to examine and analyze high or wide objects, offering: examination and analysis of high or wide objects, which do not fit to the camera field of view. Creating a large size infrared image mosaic, showing the whole object, by combining multiple infrared images; improvement of the resolution of the camera by combining multiple infrared images into a high-resolution image composite. The resulting image will be again a fully radiometric infrared image will all thermal data in it; matching remotely sensed images of the same area taken after a certain period of time, when the more recent image was probably not taken from precisely the same position; creating eye-catching collages of infrared and visual images. Image Fusion technology allows blending infrared images and digital photos together, making diagnosis and interpretation even easier. All Grayess sotware tools operate with Fluke, FLIR, NEC-Avio, Infrared Solutions, GSAT, Jenoptik, Raytek, Mikron, DIAS, Testo, AGEMA infrared cameras [31].

The Medicore IRIS-XP® [32] is real time and non-cooling infrared thermograph integrated imaging system, that offers health conditions identification and treatments assessment. It provides real time infrared image connection, real time measurement, recording and storage, patient kindly device of portable and separated camera \& console, easy and convenient camera focusing using the software, scientific interpretation ability based on academic studies, reporting and follow up analysis.

The proprietary software provided by the camera manufacturers is outlined in table 1 with the indication of type of license, operating system and features.

Table 1. The available software for thermal imaging capture and analysis per provider and type of license

\begin{tabular}{|l|l|l|l|l|}
\hline \multicolumn{1}{|c|}{ Manufacturer } & \multicolumn{1}{|c|}{ Name } & Type of License & Operating System & \multicolumn{1}{|c|}{ Files Format } \\
\hline Photometrix & CTHERM & Licensed & Windows 2000 \& XP & Proprietary (IMG \& DAT) \\
\hline FLIR & FLIR Camera Player & Freeware & Windows & Proprietarv (FLIR) \\
\hline FLIR & FLIR Quick Report & Freeware & Windows & Proprietarv (FLIR) \\
\hline FLIR & FLIR Tools & Freeware & Windows, Mac OS X & Proprietary (FLIR) \\
\hline FLIR & FLIR Tools Mobile & Freeware & iOS, Android, Kindle & Proprietary (FLIR) \\
\hline FLIR & FLIR Viewer App & Freeware & iOS & Proprietarv (FLIR) \\
\hline FLIR & FLIR Web Viewer & Freeware & Web browser & Proprietarv (FLIR) \\
\hline FLIR & FLIR Camera Player & Licensed & Windows & Proprietary (FLIR) \\
\hline FLIR & FLIR IR Monitor & Licensed & Windows & Proprietary (FLIR) \\
\hline FLIR & FLIR Reporter Pro & Licensed & Windows & Proprietary (FLIR) \\
\hline FLIR & FLIR ThermaCAM Researcher Pro & Licensed & Windows & Proprietary (FLIR) \\
\hline FLIR & FLIR ResearchIR Max & Licensed & Windows & Proprietary (FLIR) \\
\hline FLIR & FLIR Tools + & Licensed & Windows, Mac OS X & Proprietarv (FLIR) \\
\hline FLIR & FLIR Thermovision SDK & Licensed & Windows & Proprietarv (FLIR) \\
\hline FLIR & Altair & Licensed & Windows & Proprietary (FLIR) \\
\hline FLIR & Talon Ultra & Licensed & Windows & Proprietary (FLIR) \\
\hline Testo & IR Soft & Licensed & Windows & Proprietary (Testo) \\
\hline Fluke & FLUKE SmartViewer & Licensed & Windows & Proprietary (Fluke) \\
\hline Infratec & Infratec IRBIS 3 & Licensed & Windows & Proprietary (Infratec) \\
\hline Xenics & Xenics XControl & Licensed & Windows & Proprietary (Xenics) \\
\hline Xenics & Xenics Xeneth & Licensed & Windows & Proprietarv (Xenics) \\
\hline
\end{tabular}




\begin{tabular}{|l|l|l|l|l|}
\hline Xenics & Xenics Xeneth radiometric & Licensed & Windows & Proprietary (Xenics) \\
\hline Xenics & Xenics Xeneth SDK & Licensed & Windows, Linux & Proprietary (Xenics) \\
\hline Spectron & Spectron IR Software & Licensed & Windows & Proprietary (Spectron) \\
\hline Generaltools & GTi IR Report & Licensed & Windows & Proprietary (GTi, Omeqa) \\
\hline DIAS & Infrared PYROSOFT professional & Licensed & Windows & Proprietary (DIAS) \\
\hline TROTEC & Thermo Software IC Report Pro & Licensed & Windows & Proprietary (TROTEC) \\
\hline IRCameras & IRCameras Thermography Suite & Licensed & Windows & Proprietarv (IRCameras) \\
\hline Mikroptik & Smart-eye IRSee Report & Licensed & Windows & Proprietary (Omega, MO) \\
\hline Mikron Infrared & MicroView & Licensed & Windows & Proprietary (NEC, Satin) \\
\hline Mikron Infrared & MicroSpec & Licensed & Windows & Proprietary (NEC, Satin) \\
\hline Mikron Infrared & MicroSpec RT & Licensed & Windows & Proprietary (NEC, Satin) \\
\hline Med-Hot & Med-hot TotalVision & Licensed & Windows & Proprietary (FLIR) \\
\hline Grayess & Grayess IRT Cronista & Licensed & Windows & Proprietary (GAYESS) \\
\hline Gravess & Grayess IRT Analyzer & Licensed & Windows & Proprietarv (GAYESS) \\
\hline Gravess & Grayess IRT Stitch & Licensed & Windows & Proprietarv (GAYESS) \\
\hline Medicore & IRIS-XP & Licensed & Windows & Proprietary (MEDICORE) \\
\hline
\end{tabular}

\section{Discussion}

As there are no cameras designed specifically for clinical applications there is an absence of a specific complete infrared thermal imaging software for the clinical setting, which are compatible with the various capture equipment available in the market. One important aspect to take into account is to store and retrieve the images in a DICOM format [33], which is not defined for medical thermography yet. There is no doubt that there are already thermal image capture devices equipped with wired and wireless connection that satisfy the minimal requirements for clinical practice [11].

The contemporary computers and mobile devices are also equipped with compatible wireless network connection. Efforts are recommended to manufacturers, researchers and health professional to define the modality of medical thermal imaging in the DICOM standard [33]. The FLIR Tools and FLIR Tools Mobile App, apart from the record format is a good starting point. Allows the camera control and the basic image analysis features. It is worth to add the medical specific features present in the CTHERM [17] and Medicore [32] software packages and some extra features such as pre-processing tools (image enhancing), segmentation features (edge/shape detection/registration), variation analysis (spatial, temporal and frequency) and image normalization for a more accurate analysis and comparison [34]. This new software should be multiplatform to avoid usage constraints and able to be used in the emerging mobile devices and should be capable of read/import most of the proprietary file formats and convert the radiometric data with the extra needed information in the defined DICOM format.

\section{Conclusion}

A software package built dedicatedly for medical imaging applications able to be integrated with PACS and RIS systems using the DICOM standard having the latest developments in thermal imaging processing would attract more health professionals that are not aware of the importance of medical thermography, enforcing its credibility and contributing to become part of the common daily clinical practice.

\section{ACKNOWLEDGMENT}

This research is sponsored by FEDER funds through the program COMPETE - Programa Operacional Factores de Competitividade and by national funds through FCT-Fundação para a Ciência e a Tecnologia, under the Project "AAL4ALL - Padrão de Cuidados Primários para Serviços de AAL" (FCOMP-01-0124-FEDER-13852) co-financed by FEDER, Programa Operacional Factores de Competitividade.

\section{REFERENCES}

[1] Ring E.F.J., Ammer K., "Infrared thermal imaging in medicine". Physiological measurement, vol. 33.3, pp. R33, 2012.

[2] Vardasca R., Simoes R., "Current Issues in Medical Thermography", in Topics in Medical Image Processing and Computational Vision, Tavares J. M. R. S. and Jorge R. M. N. (Eds.), Springer Netherlands, pp. 223-237, 2013. 
[3] Ring E.F.J., Jones B.F., "Historical Development of Thermometry and Thermal Imaging in Medicine", Medical Infrared Imaging: Principles and Practices, M. Diakides, J. D. Bronzino and D. R. Peterson (Eds.), Chapter 2, Taylor and Francis / CRC Press, Boca Raton, USA, 2013.

[4] Plassmann P., Ring E. F. J., Jones C. D., "Quality assurance of thermal imaging systems in medicine", Thermology International, vol. 16(1), pp. 10-15, 2006.

[5] Feig S. A., Shaber G. S., Schwartz G. F., et al., "Thermography, mammography, and clinical examination in breast cancer screening. Review of 16,000 studies", Radiology, vol. 122, pp. 123-127, 1977.

[6] Ammer K., "Need for Standardisation of Measurements in Thermal Imaging". In Thermography and Lasers in Medicine, B. Wiecek (Ed.), Akademickie Centrum, Lodz (Poland), pp. 13-17, 2003.

[7] Plassmann P., Jones C. D., "Recording Thermal Images and Software Development for Standardisation of Thermal Imaging". In Thermography and Lasers in Medicine, B. Wiecek (Ed.), Akademickie Centrum, Lodz (Poland), pp. 18-24, 2003.

[8] Ring E.F.J., Ammer K., Jung A., Murawski P., Wiecek B., Zuber J., Zwolenik S., Plassmann P., Jones C.D., Jones B. F., "Standardization of infrared imaging". In Conf. Proc. IEEE Eng. Med. Biol. Soc., vol. 2, pp. 11831185,2004

[9] Schwartz R.G., "Guidelines for Neuromusculoskeletal Thermography". Thermology International, vol. 16, pp 5-9, 2006.

[10] Ammer K., "Standard Procedures for Recording and Evaluation of Thermal Images of the Human Body: The Glamorgan Protocol". Thermology International, vol. 18, pp. 125-144, 2008.

[11] Howell K.J., Smith R.E., "Guidelines for specifying and testing a thermal camera for medical applications", Thermology international, vol. 19(1), pp. 5-14, 2009.

[12] ISO TC121/SC3-IEC SC62D, "Particular requirements for the basic safety and essential performance of screening thermographs for human febrile temperature screening", 2008.

[13] ISO/TR 13154:2009 ISO/TR 8-600, "Medical Electrical Equipment- Deployment, implementation and operational guidelines for identifying febrile humans using a screening thermograph", 2009.

[14] Ledley R.S., Lusted L.B., "Reasoning Foundation of Medical Diagnosis: Symbolic Logic, Probability and Value Theory and our Understanding of How Physicians Reason", Science, New Series, vol. 130(3366), pp. 9-21, 1959.

[15] Jones B.F., Plassmann P., "Digital thermal imaging of human skin”, IEEE Engineering in Medicine and Biology Magazine, vol. 21(6), pp. 41-48, 2002.

[16] Murawski P., Jung A., Ring E.F.J., Zuber J., Plassmann P., Kalicki B., "'Image ThermaBase' - A software programme to capture and analyse thermographic images". Thermology International, vol. 13(1), pp. 5-9, 2003.

[17] Plassmann P., Murawski P., "CTHERM for standardised thermography". Thermology International, vol. 13, pp. 79, 2003.

[18] FLIR website, URL: http://www.flir.com/, last visited on 01/04/2014.

[19] TESTO website, URL: http://www.testo.com/, last visited on 01/04/2014.

[20] Fluke website, URL: http://www.fluke.com/, last visited on 01/04/2014.

[21] Infratec website, URL: http://www.infratec.de/, last visited on 01/04/2014.

[22] Xenics website, URL: https://www.xenics.com/, last visited on 01/04/2014.

[23] Spectron website, URL: http://spectronir.com/, last visited on 01/04/2014.

[24] Omega website, URL: http://www.omega.com/, last visited on 01/04/2014.

[25] DIAS infrared website, URL: http://www.dias-infrared.com/, last visited on 01/04/2014.

[26] TROTEC website, URL: http://www.trotec24.com/, last visited on 01/04/2014.

[27] IRCameras website, URL: http://www.ircameras.com/, last visited on 01/04/2014.

[28] Mikroptik website, URL: http://www.microptik.eu/, last visited on 01/04/2014.

[29] Metrum information storage website, URL: http://www.metrum.co.uk/, last visited on 01/04/2014.

[30] Med-Hot website, URL: http://www.med-hot.com/, last visited on 01/04/2014.

[31] Grayess website, URL: http://www.grayess.com/, last visited on 01/04/2014.

[32] Medicore website, URL: http://www.medi-core.co.kr/, last visited on 01/04/2014.

[33] Vardasca T., Martins H.G., Vardasca R., Gabriel J., "Integrating Medical Thermography on a RIS Using DICOM Standard", In proceedings of the XII Congress of the European Association of Thermology as Appendix 1 of the Thermology International vol. 22(3), Ammer K., Gabriel J., Vardasca R. (Eds.), Porto (Portugal), 5-8th September, pp. 79-81, 2012.

[34] Jones B. F., Plassmann P., "Digital thermal imaging of human skin", IEEE Engineering in Medicine and Biology Magazine, vol. 21(6), pp. 41-48, 2002. 